\title{
OUR Primate Roots
}

Johan M.G. van der Dennen

Department of Law, University of Groningen, Groningen, The Netherlands

j.m.g.van.der.dennen@rug.nl

A Review of the Book

The Primate Origins of Human Nature

By Carel P. Van Schaik. 2016.

Vol. 3 in the series "Foundations of Human Biology",

Edited by Matt Cartmill and Kaye Brown.

Wiley-Blackwell, Hoboken, NJ, 560 pages.

ISBN 978-0-470-14763-4 (Hardback, \$159.35)

$\underline{\mathrm{DOI}}$

Carel P. van Schaik is Professor of Biological Anthropology and Director at the Anthropological Institute and Museum of the University of Zürich, Switzerland. He is a prolific writer and one of the best researchers and theorists from the excellent school of primatologists under Jan van Hooff at the University of Utrecht.

By integrating up-to-date inter- and multidisciplinary concepts and theories from a wide variety of academic disciplines (physical and cultural anthropology, behavioral ecology, life-history theory, and comparative and evolutionary psychology), The Primate Origins of Human Nature prepares readers to understand how mammals, primates, great apes, hominids, and humans came to be. This invaluable work bridges the study of primatology and anthropology by taking a broad comparative approach, and effectively primes the reader on both primate behavioral biology and human evolution.

The Primate Origins of Human Nature is not only an invaluable resource for both students and researchers but also a creative and ground-breaking synthesis of current knowledge in the biological and social sciences that illuminates the origins and nature of humanity. It uncovers and traces primate antecedents and evolutionary causes for a broad range of supposedly unique features of human social organization, life history, and 
behavior, from aesthetics to xenophobia.

This impressive volume is a formidable tour de force. According to personal communication with the author it is written especially with psychologists and human ethologists, who are also interested in primatology, in mind. It is therefore as much a textbook, a manual and reference work, and didactic tool.

"This book is written for anyone interested in the evolution of human nature, with a basic background in natural sciences and a basic knowledge of biology, as provided in any introductory course in college or high school advanced placement. But inevitably, some readers will be more familiar with certain topics than others. They can obviously skip parts" (p.xv).

The author clearly explains, for example, the intricacies of plesiomorphic, symplesiomorphic, synapomorphic, autapomorphic, homologous, homoplastic, and analogous traits, as well as convergent evolution (concepts which will not be familiar to the average psychologist I presume).

The book contains nine sections covering all aspects of primate and hominin/human evolution, ecology and behavior. The introductory section is called "Evolution, Behavior and Culture"; Section II: "The History of Humans"; Section III: "Subsistence and Technology"; Section IV: "Sex and Sexual Selection"; Section V: "Life's Changes; Section VI: "Social Life"; Section VII: "Cooperation" (this - for myself most important - section contains chapters on cooperation in nature, warfare, morality, and religion); Section VIII: "The Cognitive Animal"; and Section IX: "Conclusions" (a preliminary synthesis of what made us human).

"Ideally, each section has a uniform structure. For each particular topic (say, sex or rearing offspring), we first introduce the general biological theory around a broad set of features, then examine their expression in the relevant nonhuman animals (primates for the shared features, selected non-primates to highlight independently derived similarities), followed by an examination of the proximate psychological mechanisms that regulate them, and finally their function and adaptive significance. Following that, we turn to humans. We must first ask whether the traits under consideration are identical or should be considered composed of different elements, each with a separate evolutionary history, mechanisms and functions. We then go through the same sequence of proximate mechanisms and adaptive significance" (p.xv).

Rather than singing its praise, or presenting and/or discussing details, I would like to highlight some surprising theoretical ideas, such as the "cooperative breeding" hypothesis (extensive allomaternal care) as the encompassing explanation for the evolution of our unique features. This grand idea, first developed by Sarah Hrdy (1999), was first forcefully presented and exposed with great clarity in a contribution in the volume Mind the Gap (2010) by Carel van Schaik and Judith Burkart. I quote the abstract of this publication: "Humans are strikingly different from our close relatives, the great apes, in mind, behavior, and life history. We propose that the evolution of these derived features was a consequence of the adoption of cooperative breeding by early Homo. Among the species that adopted it, cooperative breeding generally produced changes in psychology toward greater prosociality and greater cognitive abilities. We propose that in our ancestors, the major energetic inputs to breeding females due to 
cooperative breeding explain the derived features of human life history and lifted energetic constraints on brain enlargement. Moreover, in combination with great-apelevel cognitive abilities, the cooperative breeding psychology led to the evolution of many of the unusual socio-cognitive traits that we now celebrate as uniquely human: pedagogy, extensive cumulative culture, and cultural norms; intensive and nearly indiscriminate within-group cooperation and morality; a cooperative declarative communication system known as language; and full-blown theory of mind".

Van Schaik and Burkart surmise with some confidence that cooperative breeding arose somewhere between the earliest australopithecines and mid-Pleistocene Homo. Several lines of argument suggest that the origin coincided with the emergence of Homo erectus (or Homo ergaster) in East Africa around 1.8 Ma. First, there is evidence that these were the first hominins to acquire meat from large mammals, which necessarily indicates the presence of cooperative hunting of at least cooperative defense of large carcasses. Second, survival in the newly colonized savanna habitat presumably relied on skills acquired gradually during development, largely through social learning, involving some combination of tool-assisted hunting and processing of mammals and tool-based extraction of underground storage organs. Life on the savanna opened up the hunting niche, which is skill-intensive, and thus requires an unusually large brain as well as long learning periods and hence delayed maturation. These and other arguments would put the beginning of cooperative breeding in the period directly preceding the rise of $H$. erectus, when hominin brain sizes clearly exceeded the great ape range for the first time. Furthermore, as with other primates such as the savanna baboons, groups entering the treeless savanna are as large and cohesive as possible.

Clearly, van Schaik endorses a strong version of the savanna scenario, which for many scientists, most unequivocally Marc Verhaegen, is among the worst hypotheses ever proposed. Humans are physiological ill-adapted to dry open milieus. "Man is the opposite of a savanna inhabitant. Humans lack sun-reflecting fur, but have thermoinsulative subcutaneous fat layers, which are never seen in savanna mammals. We have a water- and sodium-wasting cooling system of abundant sweat glands, totally unfit for a dry environment. Our maximal urine concentration is much too low for a savannadwelling mammal. We need much more water than other primates, and have to drink more often than savanna inhabitants, yet we cannot drink large quantities at a time" (Verhaegen, 1987). This reasoning does not imply to say that human ancestors never lived on savannas, only that if they did, it was at the wetlands, lakes and rivers there. Apparently, we evolved running - only lately, and only about half as fast as equids, bovids, felids, or canids, and even slower than arboreal primates - in spite of our broad build, short toes and plantigrade feet, profuse sweating, and large subcutaneous fat tissues (Verhaegen, 2013: 261). Critics of the savanna evolutionary scenario are commonly ridiculed and maligned, but that does not invalidate or refute their arguments.

It is difficult for me to judge how much the cooperative breeding scenario hinges on the savanna hypothesis, but obviously it is a potential weak spot.

Equally problematic and vulnerable is van Schaik's adoption of extant foragers (huntergatherers) as models or analogues of the ancestral lifestyle, although he admits that the 
few remaining foragers tend to live in marginal habitats that may not be representative of the average historical forager (p. 79). There is a much more fundamental reason that this assumption is not warranted. Modern foragers have taken their own evolutionary trajectory and historical course. A number of anthropologists have argued that these defeated, pacified, subordinated, enclaved, and/or otherwise marginalized societies (existing nomadic forager societies) are models or analogues of human ancestral populations (the ancestral type of nomadic band), thus promoting the notion of our ancestors as paragons of 'original' peacefulness and egalitarianism. Far from being models of analogues of ancestral peoples (ethnies), however, contemporary huntergathers cannot possibly be taken as prototypical stand-ins for the human Palaeolithic way of life, as I have tried to explain in my Origin of War (1995: Ch. 7).

In spite of these (minor) criticisms, this impressive volume of condensed knowledge is highly recommended for psychologists and human ethologists. It is also an indispensable reference work for all (graduate) students in the behavioural and social sciences.

The many high-quality infographics by Perry van Duijnhoven contribute substantially to the book's informational and didactic quality.

\section{ABOUT THE AUTHOR}

Johan M.G. van der Dennen is senior researcher at the University of Groningen, the Netherlands, the author of The Origin of War (1995), as well as many publications on primate and hominid/human intergroup violence. He is a former president of ISHE.

\section{REFERENCES}

Hrdy, S.B. (1999) Mother Nature: A History of Mothers, Infants, and Natural Selection. New York: Pantheon Books.

Van der Dennen, J.M.G. (1995) The Origin of War: The Evolution of a Male-Coalitional Reproductive Strategy. Groningen: Origin Press.

Van Schaik, C.P. \& J.M. Burkart (2010) Mind the Gap: Cooperative breeding and the evolution of our unique features. In: P.M. Kappeler \& J.S. Silk (Eds) Mind the Gap: Tracing the Origins of Human Universals. Berlin: Springer Science, pp. 477-496. DOI

Verhaegen, M. (1987) Origin of hominid bipedalism. Nature, 325, 305-6. DOI

Verhaegen, M. (2013) The Aquatic Ape evolves: Common misconceptions and unproven assumptions about the so-called Aquatic Ape hypothesis. Human Evolution, 28, 3-4, 237 66. 